\title{
Anomalous field-induced effects in the sound velocity in lead magnesium niobate probed by micro-Brillouin scattering
}

\author{
S. G. Lushnikov \\ A. F. Ioffe Physical Technical Institute, Politekhnicheskaya 26, St. Petersburg 194021 Russia \\ Jae-Hyeon $\mathrm{Ko}^{\mathrm{a})}$ and Seiji Kojima ${ }^{\mathrm{b})}$ \\ Institute of Materials Science, University of Tsukuba, Tsukuba City, Ibaraki 305-8573, Japan
}

(Received 29 December 2003; accepted 19 April 2004; published online 21 May 2004)

\begin{abstract}
Field-induced changes in Brillouin scattering spectra of the $\mathrm{PbMg}_{1 / 3} \mathrm{Nb}_{2 / 3} \mathrm{O}_{3}$ relaxor ferroelectric have been examined in the vicinity of a diffuse phase transition under a dc electric field oriented along the [111] direction. It has been established that the transition into an electric-field-induced ferroelectric phase is accompanied by a step-like anomaly in the sound velocity of the quasilongitudinal acoustic phonon (QLA) propagating along the [110] direction. The changes in QLA phonon velocity at the ferroelectric phase transition were found to be either reversible or irreversible in different regions of the induced ferroelectric phase. The experimental results are compared with E-T phase diagrams. (C) 2004 American Institute of Physics.
\end{abstract}

[DOI: 10.1063/1.1760889]

$\mathrm{PbMg}_{1 / 3} \mathrm{Nb}_{2 / 3} \mathrm{O}_{3}$ (PMN) is the most intensively studied crystal in the family of relaxor perovskite-like ferroelectrics (relaxors). ${ }^{1-10}$ Interest in relaxors is due to their wide use in industry and also the possibility of investigating the lattice dynamics in partially disordered crystals. It is implied here that partially disordered crystals are those compounds in which one or several sublattices are disordered due to growth conditions or some external factor (temperature, field, etc.). In relaxors, ions with different valencies $B^{\prime}$ and $B^{\prime \prime}$ are arranged in an arbitrary manner in the $B$ positions of the perovskite lattice. In PMN, two unalike valence ions, $\mathrm{Mg}^{2+}$ and $\mathrm{Nb}^{5+}$, in a 1:2 stoichiometric ratio are in the perovskite $B$ sublattice. The PMN symmetry is cubic, and it remains cubic to helium temperatures. The ferroelectric state appears below $270 \mathrm{~K}$, where the dielectric permeability maximum is observed. The electric field applied induces a structural phase transition into the ferroelectric state in the vicinity of $210 \mathrm{~K}$ and drastically changes the PMN lattice dynamics. ${ }^{1}$

The $E-T$ phase diagram of the PMN crystal was built by Yushin for limited temperature and electric field ranges ${ }^{11}$ and then developed by Colla et al. ${ }^{12}$ In 1998, when analyzing a great amount of PMN experiments in a comprehensive review, $\mathrm{Ye}^{13}$ suggested his own $E-T$ diagram for wide ranges of temperatures and electric fields. The only common feature in these diagrams is a boundary between a paraelectric phase and ferroelectric phase with the $R 3 m$ symmetry. In all other aspects the diagrams differ considerably. A major difference is that Yushin and Ye take into account the effects of different cooling regimes (cooling under an applied electric field, cooling in the absence of an electric field but heating under an electric field, etc.), which is of fundamental importance for disordered crystals, while Colla does not do this.

The goal of our work was to study the behavior of longitudinal acoustic phonons in electric fields applied by Bril-

${ }^{a)}$ Present address: R\&D Center, Samsung Corning Co., Ltd., 472 Sin-dong, Paldal-gu, Suwon-si, Gyeonggi-do, Korea.

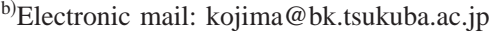

louin light scattering and to compare the obtained experimental results with $E-T$ phase diagrams.

Single crystals of PMN were grown by the Czochralski method. From a boule, a sample in the form of a parallelepiped oriented as $[111] /[1 \overline{10}] /[11 \overline{2}]$ with size $1 \times 3 \times 5 \mathrm{~mm}^{3}$ was prepared. The sample was oriented by an $\mathrm{x}$ ray. Two surfaces normal to [111] were covered with silver paste electrodes. A high-voltage power supply (HJPM-P, Matsusada Precision Inc.) was used to apply the dc electric field along the [111] direction. A high-contrast $3+3$ pass tandem FabryPérot interferometer combined with an optical microscope (Olympus BH-2) was used in micro-Brillouin experiments. Scattering was by an $\mathrm{Ar}^{+}$ion laser with a wavelength of $\lambda=514.5 \mathrm{~nm}$ and power of $100 \mathrm{~mW}$. Backscattering geometry was employed. The samples were put into a cryostat cell (THMS 6000) with temperature variations from -190 to $600{ }^{\circ} \mathrm{C}$ and stability of $\pm 0.1^{\circ} \mathrm{C}$. The free spectral range of the tandem was $75 \mathrm{GHz}$. The procedure for measurements was as follows: first the sample was cooled in zero electric field, and after a specified temperature was reached, the electric field was applied and its amplitude was varied. After this, the field was removed and the sample was warmed to room temperature. Then the cycle was repeated. In the experiments, the procedure described in Ref. 12 was used.

Studies of relative changes in the velocity of quasilongitudinal acoustic (QLA) phonons with $\mathbf{q}_{\mathrm{ph}} \|[1 \overline{1}-0]$ versus electric field applied carried out earlier ${ }^{14}$ demonstrated that the electroacoustic effect in PNM depends on the direction of the field applied. Maximum velocity changes were observed for the field applied along [111]. This dictated the choice of experimental geometry. The behavior of the acoustic phonon velocity $V$ was deduced from measurements of the shift in Brillouin frequency $\Delta \nu$ as $V=\Delta \nu \lambda /(2 n)$, where $n$ is the refractive index of the crystal. The velocity of the phonon participating in scattering is $\rho V^{2}=\frac{1}{2}\left(C_{11}+C_{12}+2 C_{44}\right)$, where $\rho$ is the crystal density, and $C_{11}, C_{12}$, and $C_{44}$ are elastic constants. Below we discuss the behavior of the pho- 


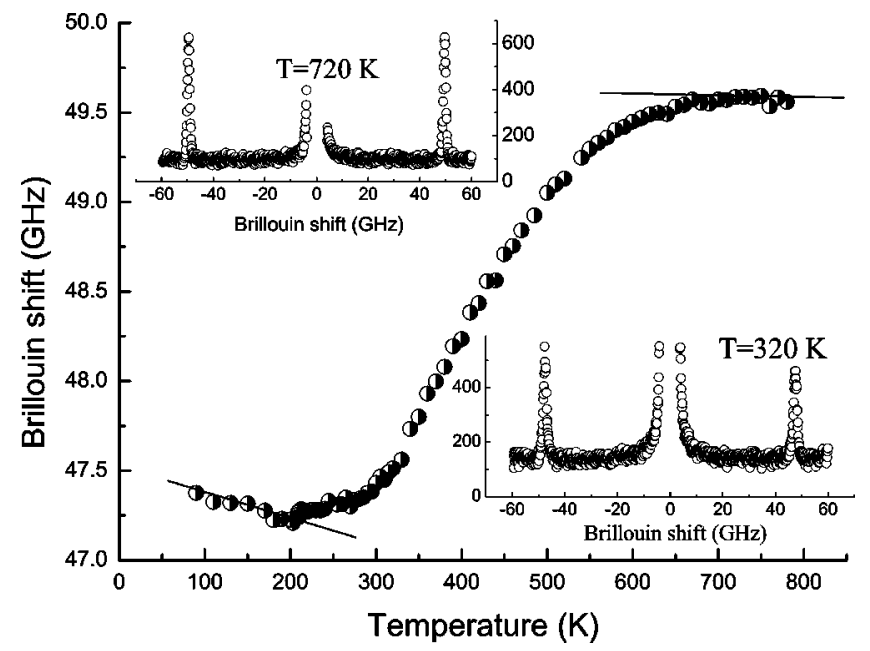

FIG. 1. Temperature dependence of the Brillouin shift in PMN crystal for a QLA phonon with $\mathbf{q} \|[110]$ in zero applied electric field. The inset shows Brillouin spectra at different temperatures.

non velocity on the assumption that $V$ and $\Delta \nu$ are linearly related.

The first step in the experiments was to study the temperature behavior of the QLA phonon with $\mathbf{q}_{\mathrm{ph}} \|[110]$ in zero applied electric field. Figure 1 shows the measured Brillouin shifts in the temperature range of $800-80 \mathrm{~K}$. It can be seen that the temperature dependence of the QLA phonon velocity agrees with typical behavior of the sound velocity of relaxors: a smooth step-like anomaly and nearly linear dependence in high- and low-temperature regions. ${ }^{14,15}$

As the next step, the behavior of the shift of frequency of the QLA phonon as a function of the electric field applied at different temperatures was investigated. At temperatures above the tricritical point $\left(T_{\text {crit }} \approx 235 \mathrm{~K}\right)$, the dependence of the phonon velocity on the applied electric field was found to exhibit two anomalies. At temperature $T=255 \mathrm{~K}$ the first anomaly is observed at $E_{t} \approx 1 \mathrm{kV} / \mathrm{cm}$, and the second occurs at about $E_{A} \cong 10 \mathrm{kV} / \mathrm{cm}$. The "return trace" of the velocity with a decrease in applied field shown in Fig. 2 by closed circles differs from the "forward trace." At the next temperature point, $T=240 \mathrm{~K}$, the dependence of the phonon velocity on the applied electric field is weaker: a small anomaly in the vicinity of $1 \mathrm{kV} / \mathrm{cm}$ and a step-like anomaly at about $E_{A} \cong 10$ $\mathrm{kV} / \mathrm{cm}$ are observed, while the hysteresis is more pronounced. The velocity anomaly in the vicinity of $10 \mathrm{kV} / \mathrm{cm}$ is likely attributable to the phase transition into an induced ferroelectric phase. Of some surprise are critical fields at which anomalies in $E_{A}$ at the temperatures discussed were observed. They are higher than the critical fields of the $E-T$ phase diagram given in Ref. 13. The reasons for this discrepancy will be elucidated.

The observed velocity anomaly of the QLA phonon at $E=E_{A}$ can be explained if we assume that there is a quadratic relation between the order parameter $(\eta)$ and deformation $(u)$ in expansion of the thermodynamic potential in the vicinity of the structural phase transition. ${ }^{16}$ In Ref. 13, observation of ferroelastic domains with the $3 m$ structure in the induced ferroelectric phase was reported. Thus, it can be supposed that the ferroelectric phase transition induced is also an improper ferroelastic transition.

Interesting behavior is exhibited by the phonon velocity Downloaded 29 Nov 2009 to 130.158.56.186. Redistribution subject

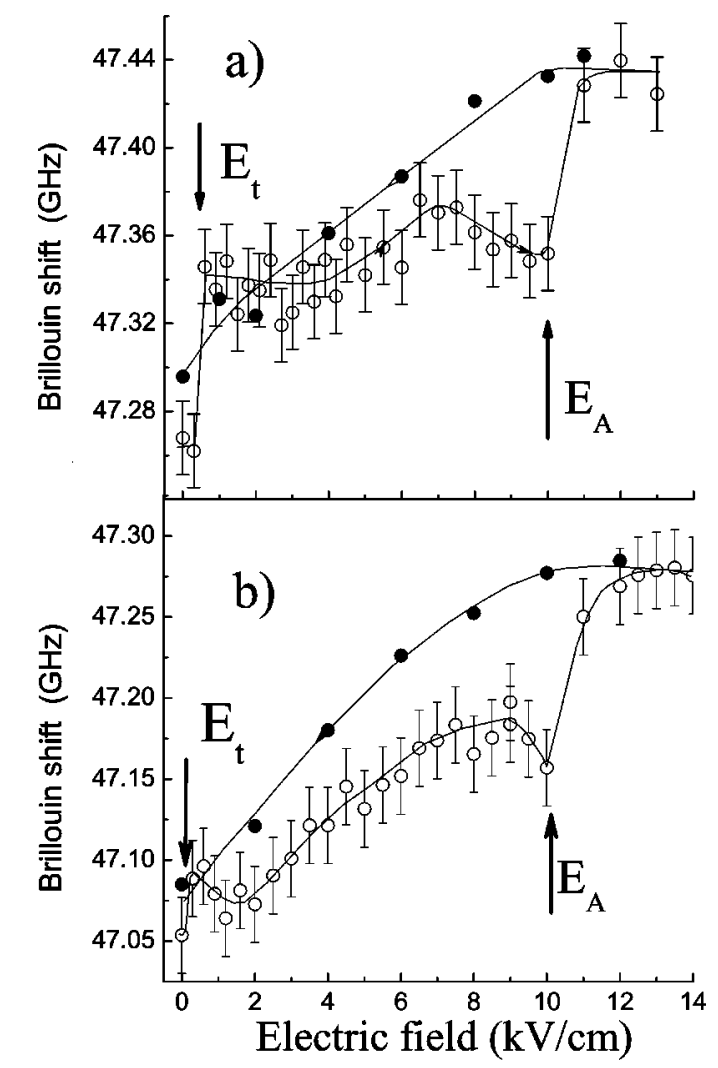

FIG. 2. Brillouin shift vs applied electric fields at different temperatures: $T=$ (a) 255 and (b) $240 \mathrm{~K}$. From here on, open and closed circles show forward and reverse changes in the electric field, respectively; solid lines are a guide to the eye.

anomaly near $E_{t}$ : it disappears in the measured $V=V(E)$ dependence as temperature decreases to $240 \mathrm{~K}$. Let us compare the dependence discussed with corresponding regions of the $E-T$ phase diagrams. ${ }^{12,13}$ It can be seen that, at low electric fields, the paraelectric and glass-like phases in the $E-T$ diagram are divided by line, C, in Colla's diagram, ${ }^{12}$ and there is no boundary between these phases in Ye's diagram. ${ }^{13}$ The velocity anomaly observed in our experiments at $E=E_{t}$ [Fig. 2(a)] corresponds to the anomaly in the vicinity of phase transformation into a glass-like phase in the $E-T$ diagram of Colla. ${ }^{12}$

The dependences measured at $T=230$ and $225 \mathrm{~K}$ were similar. It is evident from Fig. 3 that the phonon velocity remains unaltered as the electric field applied increases from 0 to $1.9 \mathrm{kV} / \mathrm{cm}$. Beginning at $2 \mathrm{kV} / \mathrm{cm}$, an increase in the field leads to a gradual growth in velocity which stops at $E \approx 4 \mathrm{kV} / \mathrm{cm}$. A further increase in the field (to $13 \mathrm{kV} / \mathrm{cm}$ ) does not affect the phonon velocity. It is well reproduced for the return trace of the applied electric field. The observed anomalies in the dependences of phonon velocity on the field applied can be attributed to an induced ferroelectric phase transition and are in good agreement with the phase boundary in the $E-T$ phase diagrams of Colla ${ }^{12}$ and of Ye. ${ }^{12}$

The situation is richer in events at $T=205 \mathrm{~K}$ (Fig. 4). This temperature region in the phase diagrams is situated below the frustrated phase transition $\left(T_{c 0} \approx 210 \mathrm{~K}\right.$ at zero field cooling). The dependence of the phonon velocity on the field applied at this temperature can be divided into four regions: from 0 to 2 and from 8 to $10 \mathrm{kV} / \mathrm{cm}$ the velocity is field independent, at $E_{A} \approx 2.2 \mathrm{kV} / \mathrm{cm}$ there is a step-like to AIP license or copyright; see http://apl.aip.org/apl/copyright.jsp 


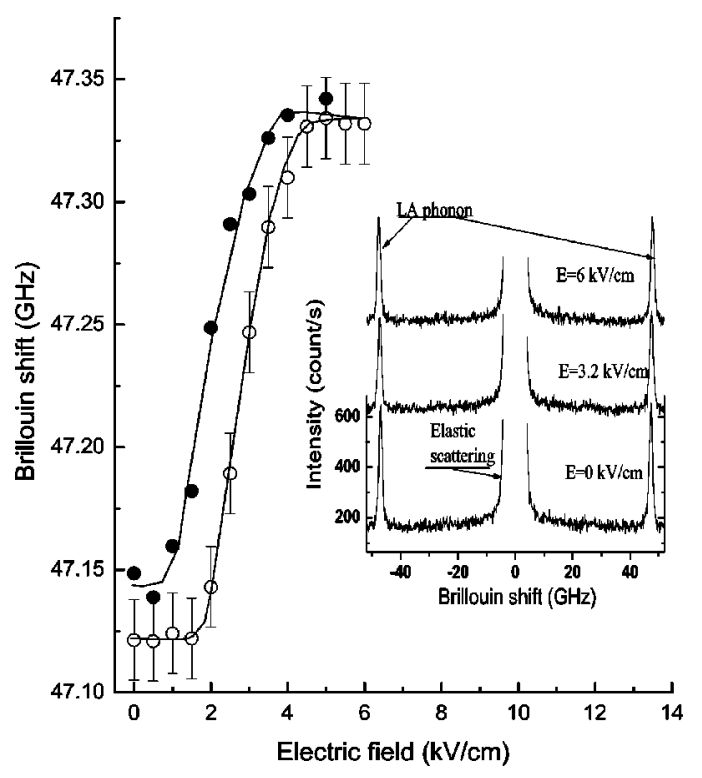

FIG. 3. Brillouin shift vs applied electric fields at $T=230 \mathrm{~K}$. The inset shows Brillouin spectra at different applied electric fields.

anomaly, and from $E_{d} \approx 3.8 \mathrm{kV} / \mathrm{cm}$ to $E=8 \mathrm{kV} / \mathrm{cm}$ the velocity slowly increases. The velocity anomaly at $E_{A}=2.2 \mathrm{kV} / \mathrm{cm}$ is consistent with the structural phase transition to an induced ferroelectric phase. This step-like anomaly (like in previous cases) can be related to invariant $\eta^{2} u$ in thermodynamic potential expansion. The situation with the anomaly in the vicinity of $E_{d}=3.8 \mathrm{kV} / \mathrm{cm}$ is more complicated. The absence of additional phases and boundaries between them in all the $E-T$ phase diagrams indicates that this anomaly is not attributable to a phase transition or transformation. Probably, this anomaly is associated with reorientational motion of polar domains in the induced ferroelectric phase of the PMN crystal. This hypothesis is supported by the fact that a decrease in applied field does not lead to changes in the phonon velocity. It is probable that the velocity will return to its initial value if the polarity of the electric field applied is reversed, as in the case of PZN-PT. ${ }^{17}$

Analysis of the obtained dependences of QLA phonon velocities on applied electric fields has shown that the phonon velocity exhibits two fundamentally different types of behavior in different regions of the $E-T$ phase diagram, i.e., (i) a reversible change in velocity when the velocity returns to its initial value when the field applied is removed (Figs. 2 and 3) and (ii) an irreversible change when the velocity does not go to its initial value if the field is removed (Fig. 4). Thus, the induced ferroelectric phase of the PMN crystal can be divided into two temperature regions, that from 255 to $225 \mathrm{~K}$ (reversible velocity changes) and that below $205 \mathrm{~K}$ (irreversible velocity changes). It can be supposed that the "true" ferroelectric state is realized in the region where changes in the sound velocity are irreversible. Note that velocity variations $(\Delta \mathrm{V})$ proved to be different in these two regions: $\Delta V$ is about $0.2 \mathrm{GHz}$ at $T=250-225 \mathrm{~K}$, and $\Delta V$ is about $0.7 \mathrm{GHz}$ around $T=205 \mathrm{~K}$, i.e., they differ by more than a factor of 3 .

To summarize, our Brillouin light scattering studies of the dependence of the velocity of quasilongitudinal acoustic

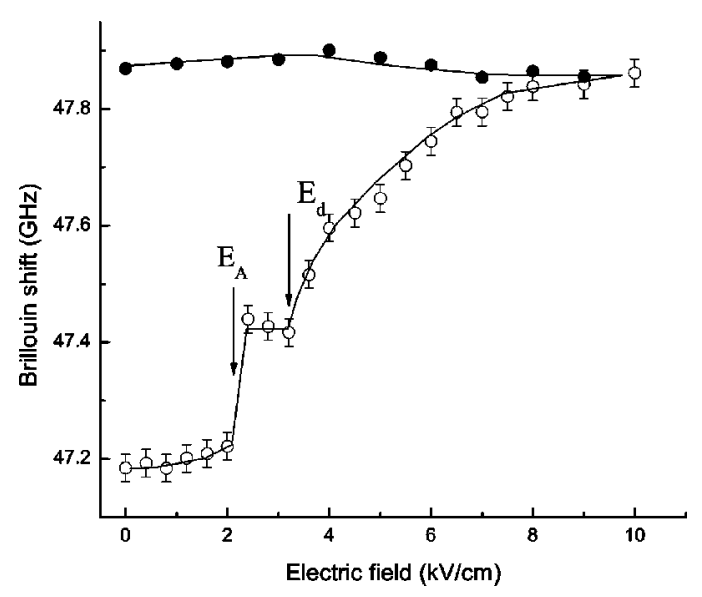

FIG. 4. Brillouin shift vs electric fields applied at $T=205 \mathrm{~K}$.

phonons on the electric field applied have shown that the induced ferroelectric phase transition is simultaneously an improper ferroelastic phase transition. Of fundamental importance is the existence of two different states in the induced ferroelectric phase with reversible and irreversible variations in the acoustic phonon velocity. It can be supposed that these states will manifest themselves in other physical properties of the PMN crystal as well. It has been found that not all our experimental data are adequately described by existing $E-T$ phase diagrams for PMN crystal.

This work was supported in part by a grant from the President of the Russian Federation, Grant No. SC2168.2003.2, and by the Russian Foundation for Basic Research, Grant No. 02-02-17678. One of the authors (S.L.) thanks the University of Tsukuba for being invited to be a visiting foreign research fellow for 2002.

${ }^{1}$ G. A. Smolenskii, V. A. Isupov, N. N. Krainik, R. E. Pasinkov, and A. I. Sokolov, Ferroelectrics and Related Materials (Gordon and Breach, New York, 1984).

${ }^{2}$ L. E. Cross, Ferroelectrics 76, 241 (1987).

${ }^{3}$ V. Westphal, W. Kleemann, and M. D. Glinchuk, Phys. Rev. Lett. 68, 847 (1992).

${ }^{4}$ I. G. Siny, S. G. Lushnikov, R. S. Katiyar, and V. H. Schmidt, Ferroelectrics 226, 191 (1999).

${ }^{5}$ R. Blinc, J. Dolinsek, A. Gregorovic, B. Zalar, C. Filipic, Z. Kutnjak, A. Levstik, and R. Pirs, Phys. Rev. Lett. 83, 424 (1999).

${ }^{6}$ H. Fu and R. E. Cohen, Nature (London) 403, 281 (2000).

${ }^{7}$ A. E. Glazounov and A. K. Tagantsev, Phys. Rev. Lett. 85, 2192 (2000).

${ }^{8}$ K. Hirota, Z.-G. Ye, Wakimoto, P. M. Gehring, and G. Shirane, Phys. Rev. B 65, 104105 (2002).

${ }^{9}$ S. N. Gvasaliya, D. Strauch, B. Dorner, S. G. Lushnikov, and S. B. Vakhrushev, Ferroelectrics 282, 21 (2003); B. Dorner, A. S. Ivanov, S. Vakhrushev, S. Lushnikov, S. Gvasaliya, D. Strauch, and K. Schmalzl, ibid. 282, 9 (2003).

${ }^{10}$ B. E. Vugmeister and H. Rabitz, Phys. Rev. B 65, 024111 (2001).

${ }^{11}$ R. Sommer, N. K. Yushin, and J. J. van der Klink, Phys. Rev. B 48, 13230 (1993).

${ }^{12}$ E. V. Colla, E. Yu. Koroleva, N. M. Okuneva, and S. B. Vakhrushev, Phys. Rev. Lett. 74, 1681 (1995).

${ }^{13}$ Z.-G. Ye, Key Eng. Mater. 155\&156, 81 (1998).

${ }^{14}$ S. G. Lushnikov and I. G. Siny, Crystallogr. Rep. 39, 675 (1994).

${ }^{15}$ J.-H. Ko, S. Kojima, and S. G. Lushnikov, Appl. Phys. Lett. 82, 4128 (2003).

${ }^{16}$ W. Rehwald, Adv. Phys. 22, 721 (1973).

${ }^{17}$ J.-H. Ko and S. Kojima, Appl. Phys. Lett. 81, 1077 (2002). 